\title{
Moderne Medien in der Volkswirtschaftslehre
}

\author{
Dieter Heyer
}

Volkswirtschaftslehre muß - wenn sie nicht zu einer reinen Dogmengeschichte verkommen soll - zugleich auch immer ein gutes Stiick „Zeitgeist" sein. Das gilt um so mehr für das Lehrgebiet „Volkswirtschaftslehre“ an Fachhochschulen der BRD. Ein solches Verständnis von Volkswirtschaftslehre als „Zeitgeist“ schließt u.a. ein, daß im Kurs „Volkswirtschaftslehre“

a) die drängensten volkswirtschaftlichen Probleme der Zeit aufgegriffen und in entsprechendem Maße behandelt werden und

\section{b) dies mit zeitgemäßen Methoden und Instrumenten} geschieht.

Nach Auffassung des Autors gibt es im Kurs ,Volkswirtschaftslehre" an Fachhochschulen der BRD zu beiden Problemkreisen dringenden Diskussionsbedarf. Die nachfolgenden Überlegungen verstehen sich als einen Beitrag zu dieser notwendigen Diskussion.

\section{zU a)}

Volkswirtschaftslehre hat sich in ihrer rund 200jährigen Geschichte als selbständige Wissenschaftsdisziplin ständig verändert. Dies konnte und mußte sie letztendlich, weil in verschiedenen Zeiträumen unterschiedliche reale volkswirtschaftliche Probleme zu ihrer Lösung drängten. Es ist kein Zufall, daß A. Smith in seinem ökonomischen Hauptwerk „Reichtum der Nationen“ der Arbeitsteilung große Aufmerksamkeit schenkte und das Wirken von J. M. Keynes wäre ohne die Weltwirtschaftskrise wohl unvorstellbar: Aus dieser Tatsache ergibt sich, daß Volkswirtschaftslehre in hohem Maße nur „aus ihrer Zeit" heraus zu verstehen ist, wie sie umgekehrt „ihre Zeit" auch immer mit geprägt hat.

Diese fuir Studium und insbesondere Lehre von Volkswirtschaftslehre existentielle Erkenntnis scheint - nach Auffassung des Verfassers - heute nicht mehr ihre notwendige Beachtung zu finden. Auf ein damit verbundenes Problem sei kurz verwiesen.

An Fachhochschulen der BRD wird das Lehrgebiet „Volkswirtschaftslehre“ auch gegenwärtig - letztlich auf J. M. Keynes zurüickgehend - in der klassischen Weise als Mikro- und Makroökonomie sowie Außenwirtschaftstheorie studiert und gelehrt. Diese eher traditionelle Art - der sich offensichtlich auch viele Autoren von Lehrbüichern zur Volkswirtschaftslehre verpflichtet fühlen - der Lehre und des Studiums von Volkswirtschafts- lehre entspricht - nach Überzeugung des Autors - nicht mehr dem notwendigen „Zeitgeist“.

Insbesondere die Anfang der 90er Jahre einsetzende Globalisierung der Wirtschaft und Internationalisierung der Produktion, deren volkswirtschaftlichen wie gesamtgesellschaftlichen Folgen heute nur bedingt absehbar sind, erfordern ebenso wie die seit Jahren anhaltende rasche Entwicklung des internationalen Handels auch und gerade im Kurs „Volkswirtschaftslehre“ eine weitaus stärkere Beachtung. Dafür bieten gegenwärtig weder der für die Außenwirtschaftstheorie vorgesehene Zeitrahmen noch der Platz der Außenwirtschaftstheorie innerhalb der Volkswirtschaftslehre notwendige Bedingungen.

Dem Autor sind die methodologischen Probleme einer Integration der Außenwirtschaftstheorie in die Mikround Makroökonomie durchaus bewußt, dennoch sollten die in diese Richtung zielenden Bemuihungen und Diskussionen weitergefuihrt werden. Ohne Einbeziehung außenwirtschaftlicher Aspekte verfehlt heute eine Markt- und Preistheorie ebenso wie eine Konjunkturoder Geldtheorie weitestgehend ihr Ziel. So lange die o. g. methodologischen Probleme nicht gelöst sind, sollte der Außenwirtschaftstheorie im Rahmen des Kurses „Volkswirtschaftslehre“ an Fachhochschulen der BRD ein größeres Zeitbudget zur Verfügung gestellt werden. Dies muß nicht zu einer Erhöhung der für die Volkswirtschaftslehre insgesamt zur Verfügung stehenden Stundenzahl fuihren, wenn die in der Mikorökonomie vermittelten Lehrinhalte ernsthaft hinsichtlich ihrer methodologischen und praktischen Relevanz überprüft werden. Eine spürbare Verstärkung außenwirtschaftlicher und internationaler Aspekte im Rahmen des Studiums und der Lehre von Volkswirtschaftslehre scheint dringend geboten, wenn auch Studierende Volkswirtschaftslehre als „Zeitgeist" erleben und begreifen sollen.

\section{zu b)}

Zeitgemäßes Studium der Volkswirtschaftslehre schließt heute auch die Nutzung moderner Medien ein. Dabei geht es insbesondere um die Nutzung moderner Medien zur Informationsbeschaffung sowie zur Unterstiitzung des Studiums in Form von Simulation volkswirtschaftlicher Prozesse bzw. als interaktive Lernmodelle. Insbesondere das Internet bietet den Studierenden für die Anfertigung von Beleg- und Diplomarbeiten eine Fülle von Informationen, die sich i. d. R. durch einen hohen Grad an Aktualität auszeichnen. Außerdem sind sie zu jeder Zeit und ohne nennenswerte Kosten be- 
schaffbar. Aus langjähriger Lehrtätigkeit ist dem Autor bekannt, daß vor allem Studierende in den ersten Semestern zum Teil nur zögerlich auf das Internet als hervorragende Möglichkeit zur Informationsbeschaffung zurückgreifen. Deshalb sollten alle Hochschullehrer Studierende - auch bereits in den ersten Semestern - noch stärker zur Nutzung des Internet motivieren.

Weitaus komplizierter stellt sich die Situation bei der Nutzung moderner Medien zur Simulation volkswirtschaftlicher Prozesse bzw. als interaktives Lernmedium dar. ${ }^{1}$ Obwohl am Markt eine Vielzahl von Programmen angeboten wird, scheint ihre Einbeziehung in Studium und Lehre der Volkswirtschaftslehre an Fachhochschulen nur zögerlich voranzukommen. ${ }^{2}$ Dabei sind die Vorteile der Nutzung moderner Medien zur Simulation volkswirtschaftlicher Prozesse bzw. als interaktive Lernmodelle unïbersehbar.

Zu ihnen gehören u.a.:

- Tempo, Intensität, Umfang und optimale Studienzeit sowie -dauer können von den Studierenden weitestgehend selbst bestimmt werden;

- Entwicklung des abstrakten und interdisziplinären Denkens;

- Training von Entscheidungssituationen;

- Entwicklung des Denkens in volkswirtschaftlichen Zusammenhängen.

Natürlich gibt es auch bei der Nutzung moderner Medien in Lehre und Studium der Volkswirtschaftslehre nicht den Königsweg, das Patentrezept. Ihre Nutzung muß den zu behandelnden inhaltlichen Fragen und Problemstellungen letztlich untergeordnet werden.

Ihr Einsatz kann auch - zumindest latent - ein von der Wirklichkeit abgehobenes mechanizistisches Weltbild befördern. Dennoch sollten wir uns vor dem Hintergrund o. g. Vorteile in verstärktem Maße mit dem Einsatz moderner Medien in Lehre und Studium der Volkswirtschaftslehre auseinandersetzen. Dabei gilt es auch die guten Erfahrungen anderer Hochschulen, z. B. der Pädagogischen Hochschule Freiburg oder der Universität Tübingen, für die TFH Wildau zu erschließen.

\section{Literafur}

[1] vgl. u.a.: Studienhilfe Computer. Simulation volkswirtschaftlicher Modelle. Wirtschaftswissenschaftliches Studium. H. 12, 1993, S. 633 ff; W. Lorenz, C. Lange, IS-LM-Analyse auf dem PC. Wirtschaftswissenschaftliches Studium. H. 11, 1995, S. 593 ff; C. Trumpf, Computergestiitztes Lernen in der Wirtschaftslehre: Allgemeine Überlegungen und kritische Analyse eines Beispiels, www.ph-freiburg.de/ wirtscha/construm.htm; www.studium-fhb.purespace.de/ oefvwl.htm

[2] F. Schanda, Computer Lernprogramme: wie damit gelernt wird; wie sie entwickelt werden; was sie im Unternehmen leisten. Weinheim, Basel, 1995; S. Seufert, Computer Assisted Learning (CAL). Grundlagen, Varianten, Entwicklung., Deutscher Universitätsverlag. Wiesbaden 1996

\section{Autor}

Dr. sc. phil. et. Dr. oec. Dieter Heyer

Technische Fachhochschule Wildau

Fachbereich Betriebswirtschaft/Wirtschaftsinformatik

Bahnhofstraße

15745 Wildau

Tel. (0 33 75) 508-910 